\title{
A PRACTICAL APPROACH TO EVALUATE THE MEASUREMENT UNCERTAINTY BUDGET FOR CALIBRATION OF STRAIN GAUGE BASED HIGH-PRECISION CARRIER FREQUENCY AMPLIFIERS
}

\author{
Marco M. Schäck ${ }^{1}$ \\ ${ }^{1}$ Hottinger Brüel \& Kjær GmbH (HBK), Darmstadt, Germany, marco.schaeck@ hbkworld.com
}

\begin{abstract}
:
For high-precision measurements of strain gauge-based transducers, $225 \mathrm{~Hz}$ carrier frequency measuring amplifiers are primarily used [1]. The benefits of this carrier frequency method were discussed in previous publications. This publication shows the measurement uncertainty that can be achieved by calibrating an amplifier based on this method. Possibilities for improving the measurement uncertainty and the physical limit from the user's point of view are shown.
\end{abstract}

Keywords: measurement uncertainty, strain gauge, carrier frequency, physical limit, auto- and background-calibration

\section{INTRODUCTION}

The true value of something cannot be measured because every measurement contains errors. A measurement is therefore only complete when an estimation of the measurement uncertainty is assigned to each measured value [2]. This is essential, especially when calibrating high-precision measuring amplifiers. It is therefore important to understand the measurement uncertainty related to a calibration of a strain gauge-based amplifier. In this paper, a practical uncertainty for calibrating a high-precision strain gauge-based carrier frequency amplifier. By using a BN100A bridge standard, the calibration of an MX238B precision amplifier of the HBM QuantumX product family with a specified class accuracy of only $0.0025(25 \mathrm{ppm})$ is considered in detail [3]. The influencing parameters for the measurement uncertainty calculation are listed. The exact order of magnitude and probability distribution are determined, to obtain a resulting overall measurement uncertainty. Finally, the largest uncertainty contributions in the overall uncertainty budget are considered in order to identify possible optimisations.

\section{CALIBRATION SETUP}

Before calibrating a measuring amplifier, it is necessary to precisely define the measuring setup. If possible, influencing factors regarding the measurement uncertainty should be eliminated or reduced as much as possible. The most accurate bridge calibration standard currently commercially available worldwide, the HBM BN100A, is therefore used to calibrate the high-precision amplifier MX238B. Figure 1shows the calibration setup with the used components.

First, a calibration certificate is required for the
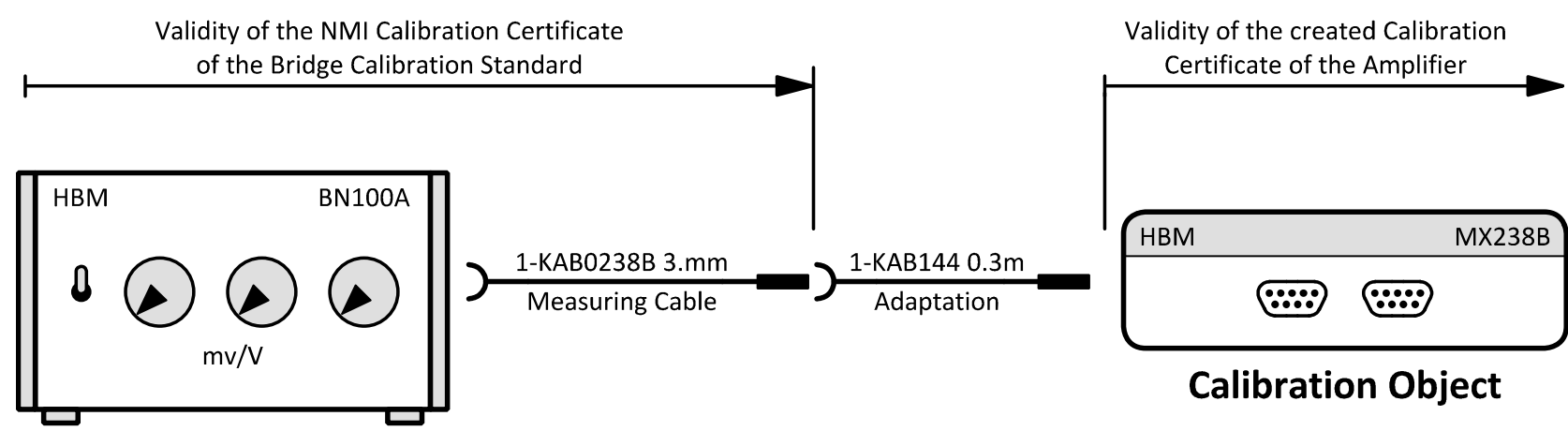

\section{Calibration Standard}

Figure 1: Setup of measurement for calibration of the MX238B approach is shown to determine the measurement

traceability for the individual bridge calibration 
standard. A direct traceability to a national reference standard at a national metrology institute (NMI) generally provides the best available uncertainty [4] Alternatively, the used bridge standard can also be traced back to a calibration laboratory (e.g. DKD German Calibration Service), which is directly connected to the national metrological institute. The possible measurement uncertainty is in this case greater with respect to the uncertainty of the NMI. If the calibration of the bridge calibration standard also includes the necessary measuring cable (1-KAB0238B), then this is also included in the specified measurement uncertainty in the calibration certificate. The calibration certificate is in this case valid for the end of the measuring cable. The used bridge standard in the measurement setup is connected to the measuring amplifier with an adapter (1-KAB144).

Boundary conditions of the measurement, including the ambient temperature and humidity, must also be defined. For this exemplary consideration, default workplace conditions at $\mathrm{HBM}$ are assumed. It is assumed that the temperature can vary between $19{ }^{\circ} \mathrm{C}$ and $26^{\circ} \mathrm{C}$ during a complete year. These environmental conditions are continuously monitored. The measurement is only carried out after all boundary conditions are met and a steady state has been reached within the specified tolerances.

\section{CONSIDERED UNCERTAINTY COMPONENTS}

After defining the calibration setup, the factors that may cause unwanted changes or variations to the measurement results must be identified. Figure 2 shows the influencing uncertainty contributions divided into three main groups, namely calibration standard, calibration object and calibration method.

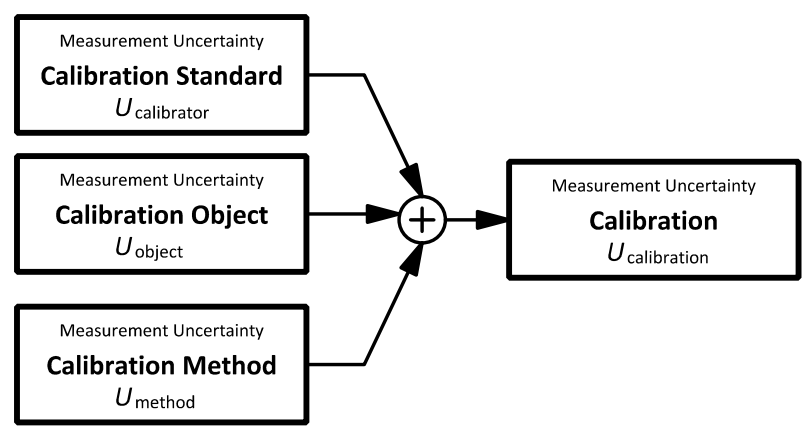

Figure 2: Grouping of the uncertainty contributions

The process to define the uncertainty model is not always easy, because any influences can affect a measurement result. A useful way to visualise how different sources of measurement error contribute to the overall measurement result is the Ishikawa diagram. A first step is to evaluate the information in the technical data sheets of the devices involved in the calibration [5][6]. If some influencing factors cannot be found in the data sheets, they must be measured individually or requested from manufacturers. Influencing factors must never be neglected. Figure 3 shows a simplified diagram with the influencing parameters when calibrating a high-precision measuring amplifier.

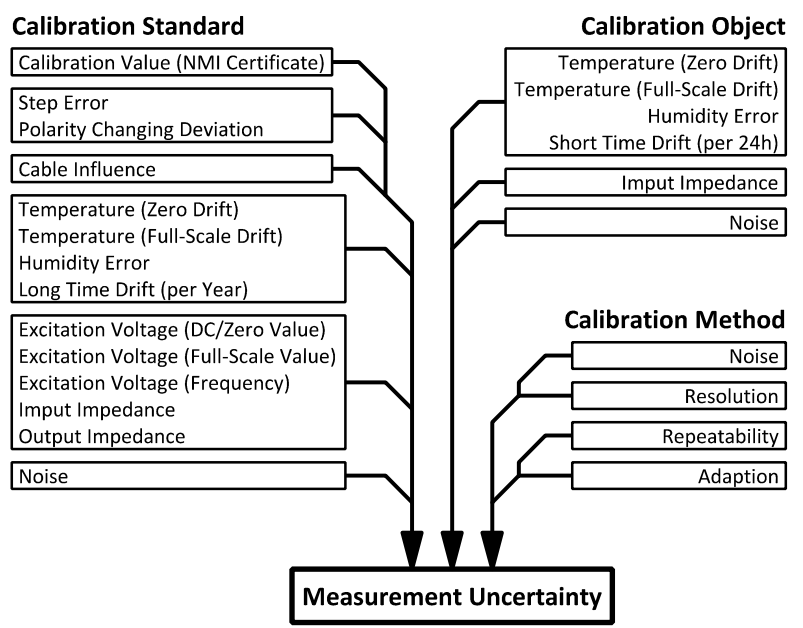

Figure 3: Ishikawa diagram for the measurement uncertainty

\subsection{Calibration Standard}

The calibration standard is the reference for the calibration which is traced back to national standards. Regarding the calibration of a high-precision strain gauge-based amplifier, one main influencing factor will be the measurement uncertainty of the calibration certificate of the NMI for the bridge calibration standard. The expanded measurement uncertainty of the calibration certificate for a BN100A carried out by the PTB (Physikalisch-Technische Bundesanstalt, Ratio and Sampling Techniques, Working Group 2.12, Germany) is indicated with $11 \mathrm{nV} / \mathrm{V}$ (4.4 ppm) for a nominal value of $2.5 \mathrm{mV} / \mathrm{V}$ and an excitation voltage of $5 \mathrm{~V}_{\text {rms. }}$. It is mentioned that the measurement uncertainty specified in calibration certificates is usually including an expansion factor $k$ of 2 and corresponds to a normal distribution.

If there are explicit calibration values in the calibration certificate for all used steps and polarity combinations of the bridge calibration standard, a step and polarity error has not to be considered. Likewise, the measurement cable has also not to be considered in the measurement uncertainty, if the calibration certificate is valid at the end of the measurement cable. In this way, these uncertainty factors can be eliminated. If the measuring cable is not considered in the calibration, the influence can be estimated based on a previous publication [7].

Another influencing group regarding the uncertainty of the calibration standard is the drift and stability. Without explicit specification in the data sheet, the HBM class accuracy of the bridge calibration standard BN100A of 0.0005 applies to zero point and full-scale values (in this case 
$<5 \mathrm{ppm} / 10 \mathrm{~K}$ each). Regarding the humidity and long-term stability, for which the error is depends on the calibration interval, no information is given in the data sheet. The humidity influence can be found in publications from the PTB [8]; internal HBM measurements show a very similar behaviour.

The influence of the humidity from $35 \%$ to $80 \%$ at $22{ }^{\circ} \mathrm{C}$ is very low (non-condensing) and assumed to be less than $6 \mathrm{nV} / \mathrm{V}(<2.4 \mathrm{ppm})$ The maximum relative humidity and temperature effect is considered to be very low, which means that the device is very stable under relative humidity or temperature changes. The outstanding long-term stability of the BN100A is assumed to be less than $5 \mathrm{nV} / \mathrm{V}(<2 \mathrm{ppm})$ per year. Long-term stability data can be found in previous publications [9][10][11].

The influences of the bridge excitation voltage and the source and sink impedances are negligible and mentioned only for completeness [12]. For example, a slight DC component has no influence on the measurement result due to the carrier frequency method [13][14][15], and a variation of the bridge excitation voltage by a few percent has no measurable influence $(<2.8 \mathrm{nV} / \mathrm{V}$ per volt of excitation voltage) on the output signal $(\mathrm{mV} / \mathrm{V})$ of the bridge calibration standard.

Due to the active regulation of the individual divider stages of the bridge calibration standard the frequency response is relatively flat and the phase nearly zero over a very wide frequency range. Figure 4 shows a rough overview of the amplitude and phase response of the BN100A on a logarithmic scale in $\mathrm{dB}$.

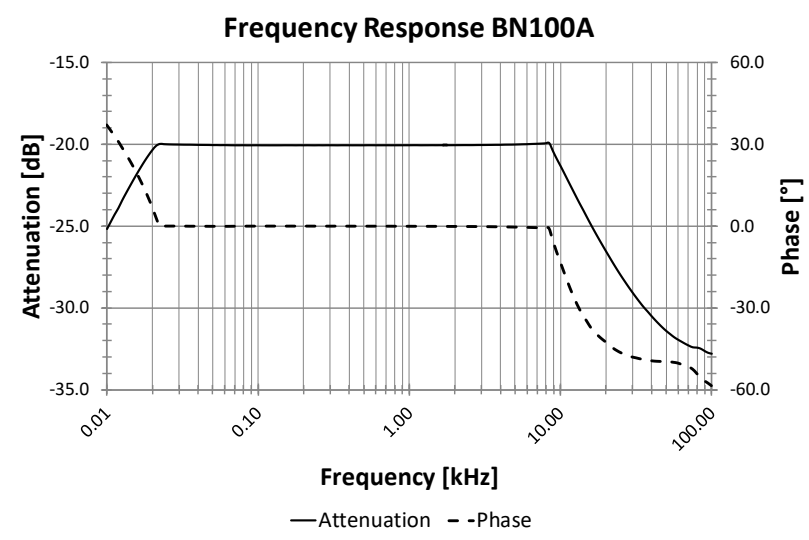

Figure 4: Frequency response of the BN100A

To determine the exact frequency dependency in the ppm range around the carrier frequency, measurements were carried out with a modified carrier frequency measuring amplifier MX238B. With this modified amplifier, the carrier frequency can be changed in fine frequency increments. Slight possible frequency variation of the bridge excitation voltage around the carrier frequency of $225 \mathrm{~Hz}$ has no effect to the calibration signal output, even in the ppm range.
For $225 \mathrm{~Hz}$ carrier frequencies outside the normal frequency range of the carrier frequency (other carrier frequencies e.g. $600 \mathrm{~Hz}$ ) the BN100A must be individually modified and at least be tuned to resonance (BN100A is only specified for $225 \mathrm{~Hz}$ ).

A final important aspect is the noise of the bridge calibration standard. The noise of the BN100A, which is very low by design, is not specified in the data sheet and it is very difficult for users to measure it (separate the noise of the bridge calibration standard and the noise of the amplifier). A comparison of a passive $350 \Omega$ full bridge transducer with the BN100A for a supply of $5 \mathrm{~V}_{\text {rms }}$ and a detuning of $2.5 \mathrm{mV} / \mathrm{V}$ shows no difference on the MX238B in terms of noise. In any case, it is not important for the measurement uncertainty of the calibration whether the noise comes from the bridge calibration standard or from the measurement amplifier. The total noise is of importance. This can simply be determined in the complete measuring chain. Therefore, the total noise is not considered as individual components, but is assigned later to the calibration process as a sum.

\subsection{Calibration Object}

In comparison to the bridge calibration standard, the number of parameters to be considered for the measuring amplifier is significantly lower (only if the amplifier is the object to calibrate). The temperature and humidity influence on the MX238B measuring amplifier is very low. The temperature and humidity are not important for the calibration uncertainty and are in the calibration certificate only for documentation. Because of the excellent data from the measuring amplifier, due to the low sensitivity coefficient (partial derivative), an uncertainty in the measurement of temperature and humidity (external sensor, traced back) would not make a meaningful contribution to the measurement uncertainty.

The long-term stability of the measuring amplifier is also not important for its calibration. The measuring amplifier must be stable during the calibration measurement, which is guaranteed by the integrated and patented background calibration of the MX238B after the running in phase [16][17].

However, it must be checked whether the input impedance of the measuring amplifier influences the calibration signal of the bridge calibration standard. Taking into account the impedances (output impedance of the bridge standard, input impedance of the measuring amplifier), the conclusion is that the input impedance has no influence to the calibration signal, due to the very high-impedance of the measuring amplifier input [18].

The noise of the amplifier specified in the data sheet is irrelevant, because the noise is assigned to the calibration process as total noise. 


\subsection{Calibration Method}

The final group, the calibration method parameters, includes the total noise, consisting of the noise of the calibration standard and the measuring amplifier. Because no information for the noise is available for this special measuring chain, the total noise must be determined for this application by separate measurement.

It is easy to determine the noise for the worst calibration point and then use this result as an uncertainty contribution for all other calibration points. The worst case is the largest calibration value of the measuring range. In this case, the noise contains not only the noise of the measuring amplifier input, but also noise of the bridge excitation voltage, the reference voltage of the analogue-digital converter of the amplifier and the noise of the active circuit electronics of the bridge calibration standard.

Ideally, these additional contributions are not significant and the noise is independent of the calibration value (constant over the complete measuring range). Figure 5 shows the noise over time for the calibration setup.

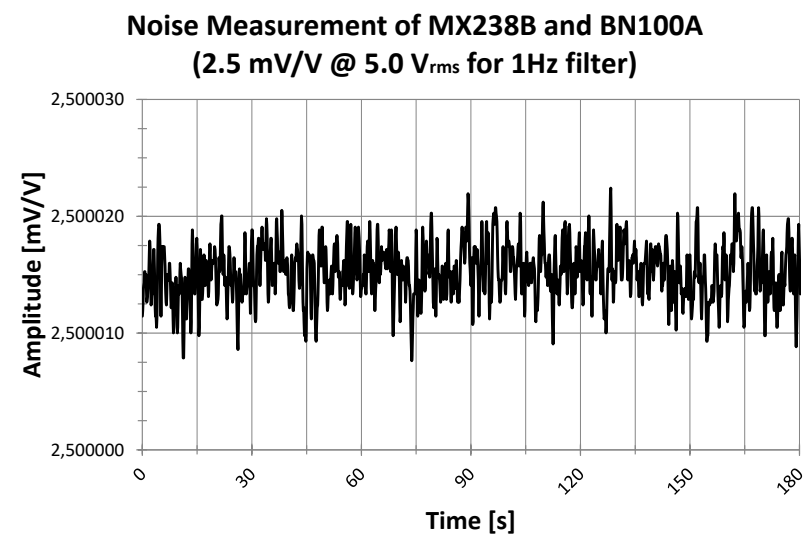

Figure 5: Noise measurement for a combination of MX238B and BN100A

To determine the noise, a measurement is recorded over a sufficiently long interval with a defined low-pass filter (e.g. Bessel $1 \mathrm{~Hz}-3 \mathrm{~dB}$ ). This is the filter used for calibration of the amplifier. The standard deviation $\sigma$ is to be determined from the recorded measured values and is in this case $2.3 \mathrm{nV} / \mathrm{V}(0.9 \mathrm{ppm})$ [19]. The standard deviation corresponds to the effective noise of the complete measuring chain and can be used for the measurement uncertainty analysis.

The hypothesis is made that the noise is normally distributed. This hypothesis must be checked by determining a histogram or by a statistical test for normal distribution. Due to the low cut-off frequency of the filter and the associated very low noise of the measured value the measured value has to be filtered digitally to get a higher amplitude resolution in a post process to have a higher bin resolution in the histogram. Otherwise, the noise cannot be displayed with a sufficient bin resolution for the histogram. Figure 6 shows the histogram of the noise for a modulation of the bridge calibration standard of $2.5 \mathrm{mV} / \mathrm{V}$.

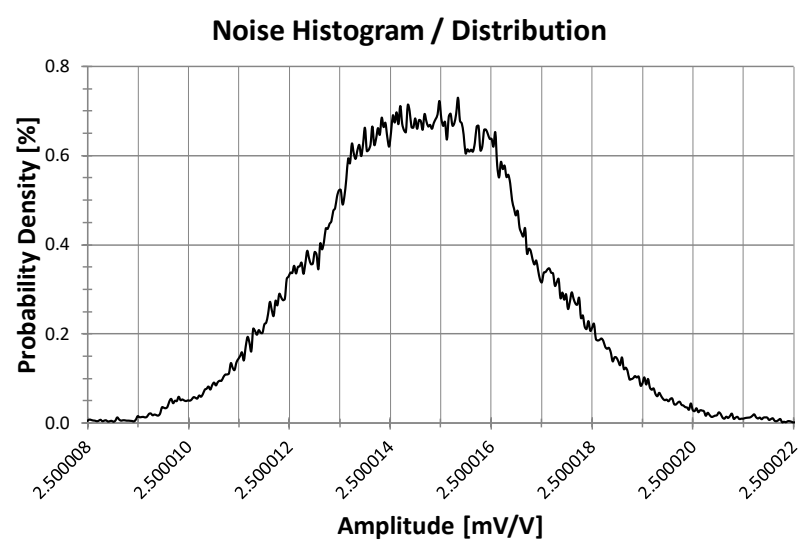

Figure 6: Noise histogram for a combination of MX238B and $\mathrm{BN} 100 \mathrm{~A}$

Similar to the case of noise, there is no information for the reproducibility for this special application. The considered MX238B precision measuring amplifier uses the proven $225 \mathrm{~Hz}$ carrier frequency method and a six-wire circuit. The advantage of this method has been explained in a previous publication [20]. Influences such as thermal/contact voltages due to different materials of the pins, other offset effects and contact resistances on the connectors are suppressed.

The carrier frequency method also enables the use of temperature-stable and long-term-stable inductive bridge standards, such as the BN100A bridge calibration standard. Even with the best $225 \mathrm{~Hz}$ carrier frequency technology and the use of the six-wire circuit, there must be a check of how reproducibly identical measurements are. For this, several identical measurements are repeated under the same boundary conditions (in this case fifty independent measurements). Comparable to the noise measurement, the type of distribution must also be determined for the reproducibility measurement. Figure 7 shows the distribution of the repeatability. This uncertainty contribution results in a normal distribution with an uncertainty of $1 \mathrm{nV} / \mathrm{V} \quad(0.4 \mathrm{ppm})$. The repeatability test is performed once, not for every calibration. For a generalised sampling repeatability data are collected and the result is used for all similar subsequent calibrations. 


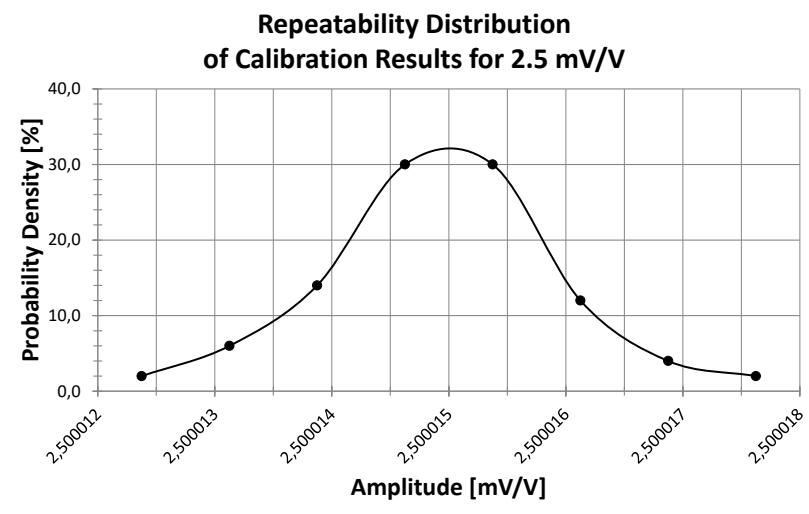

Figure 7: Repeatability distribution of calibration results

As previously mentioned, the calibration certificate for the bridge standard is valid at the cable end of the bridge standard. Adapter cables are often necessary for the current plug-in system of the measuring amplifier. The influence of the adaptation must be determined for this. This can be estimated by measuring several cascaded adaptations. Measurements in this scenario showed no significant effects for the required adaptation (1-KAB144).

\section{COMBINED MEASUREMENT UNCERTAINTY}

The complete measurement uncertainty parameters have been collected in a table with the corresponding distribution type. The considered measurement uncertainty components are uncorrelated, therefore the geometric addition of these measurement uncertainty components gives the total measurement uncertainty (combined all individual A and B types of uncertainties), as shown in equation (1).

$$
\begin{gathered}
U=k \cdot \sqrt{\sum U_{\text {calibrator }^{2}}+\sum U_{\text {object }^{2}}+\sum U_{\text {method }}^{2}} \\
=16.8 \frac{\mathrm{nV}}{\mathrm{V}} \cong 6.7 \mathrm{ppm}
\end{gathered}
$$

If this is expanded by the factor $k$ of 2 , the expanded measurement uncertainty is obtained. A coverage factor of $k$ equal to 2 would indicate a confidence of approximately $95 \%$.

\section{CONCLUSIONS}

In this paper, all the main factors influencing the calibration uncertainty of a $225 \mathrm{~Hz}$ precision carrier frequency measuring amplifier were discussed. Mainly due to the carrier frequency method and the six-wire circuit, only a few factors can (significantly) influence the measurement uncertainty.

Figure 8 shows the main influencing factors previously explained based on the measurement uncertainty amounts. The largest uncertainty contributions are of a very similar order of magnitude. This includes the temperature effects.
The temperature and humidity effects can be further reduced by a more precise climatic environmental condition, as in calibration laboratories.

The long-term stability used in a measurement uncertainty analysis can be based on the class accuracy of the bridge standard BN100A. This effect of the long-term stability of the bridge calibration standard BN100A could also be determined for an individual device and, if necessary, a recalibration could be done in a smaller interval. This measurement uncertainty contribution could thus be reduced in the uncertainty analysis. Measurements have shown that the long-term stability of this bridge calibration standard is typically significantly lower than the value specified in the data sheet.

Parts of the Measurement Uncertainty

(Calibration point $2.0 \mathrm{mV} / \mathrm{V}$ 5Vrms $1 \mathrm{~Hz}$ Bessel Filter)

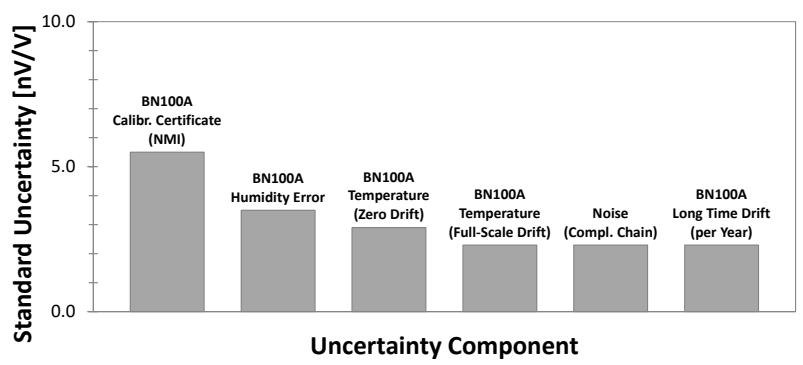

Figure 8: Parts of the measurement uncertainty

The noise of the entire measuring chain can also be further reduced by changing the digital low-pass filter. The noise is expected to reduce with the square root of the bandwidth (valid for white noise).

Since all influencing factors of the measurement uncertainty are uncorrelated and therefore are added geometrically, possible optimisations do not lead to a significant reduction in the overall measurement uncertainty. A large reduction is not possible due to the given measurement uncertainty in the calibration certificate of the bridge standard BN100A. If it would be possible to eliminate all influencing factors except for the measurement uncertainty of the calibration certificate of the bridge calibration device, the measurement uncertainty could only be reduced by a maximum factor of around 1.5. Optimisations only marginally improve the overall measurement uncertainty. This shows that the $225 \mathrm{~Hz}$ precision carrier frequency measuring amplifier MX238B from HBM measures at close to the achievable limit. A new measurement uncertainty analysis with other influencing factors must be created to consider a measuring chain out of a transducer and measuring amplifier.

\section{REFERENCES}

[1] K. Hoffmann, An Introduction to Measurements using Strain Gages, HBM, Darmstadt, Germany, pp. 171-179, 1989. 
[2] Joint Committee for Guides in Metrology (JCGM/WG 1), "Guide to the Expression of Uncertainty in Measurement" (GUM), 2008.

[3] M. Kreuzer, "Kalibrieren des Digitalen PräzisionsMeßgeräts DMP39 mit einem speziellen Brückennormal", Messtechnische Briefe (HBM) issue 17, Darmstadt, Germany, pp. 67-73, 1981.

[4] G. Ramm, "Kalibrieren von Brückennormalen für die Dehnungs-messstreifen-Messtechnik", Messtechnische Briefe (HBM) issue 24, Darmstadt, Germany, pp. 10-16, 1988.

[5] Hottinger Baldwin Messtechnik GmbH, Datasheet BN100A.

[6] Hottinger Baldwin Messtechnik GmbH, Datasheet MX238B.

[7] M. Schäck, "Underestimated Impact of Measuring Cables on High-Precision Carrier Frequency Amplifier Results and Compensation Methods therefor", Proceedings of XXI IMEKO World Congress, Prague, Czech Republic, 2015.

[8] K. Khaled, D. Röske, (eds.), "Humidity and temperature effects on torque transducers, bridge calibration", Measurement; 2015.

[9] A. Schäfer, H. Kitzing, "DMP41 - A new chapter of ultra-precision instrument for strain gauge transducers", Proceedings of XX IMEKO World Congress, Busan, Rep. of Korea, 2012.

[10] A. Schäfer, "A novel type of precision amplifier for strain gauge based transducers", Proceedings of TC4 International Conferences IMEKO Congress, Budapest; Hungary, 2016.

[11] A. Schäfer, "The Ultra-Precision Instrument DMP41 - First Experiences \& Appropriate Filter Settings"; Proceedings of TC22 International Conferences IMEKO Congress, Cape Town, Republic of South Africa, 2014.

[12] L. Rafflenbeul, M. Schäck, R. Werthschützky, "Optimization of the input impedance of a lownoise unipolar powered amplifier", Proceedings of Eurosensors XXV, Athens, Greece, 2011.

[13] M. Schäck, "High-Precision Measurement of Strain Gauge Transducers at the Physical Limit without any Calibration Interruption", Proceedings of TC3 International Conferences IMEKO Congress, Cape Town, Republic of South Africa, 2014.

[14] E. Heringhaus, "Trägerfrequenz- und Gleichspannungs-Meßverstärker für das Messen mechanischer Größen - ein Systemvergleich aus anwendungstechnischer Sicht - Teil 1: Arbeitsweisen und Vergleich charakteristischer Eigenschaften", Messtechnische Briefe (HBM) issue 18, Darmstadt, Germany, pp. 42-49, 1982.

[15] E. Heringhaus, "Trägerfrequenz- und Gleichspannungs-Meßverstärker für das Messen mechanischer Größen - ein Systemvergleich aus anwendungstechnischer Sicht - Teil 2: Verhalten gegenüber externen Störeinflüssen und praktische Auswahlhilfen", Messtechnische Briefe (HBM) issue 18, Darmstadt, Germany, pp. 70-73, 1982.

[16] M. Schäck, H. Kitzing, "Messverstärker mit Hintergrundjustierung und Verfahren dafür", DE 102013014876 B3, German Patent and Trade Mark Office, Munich, Germany, 2014.

[17] M. Schäck, "Long Term Proven and Optimized High-Precision $225 \mathrm{~Hz}$ Carrier Frequency Technology in a Modern and Universal Data Acquisition System", Proceedings of TC3 International Conferences IMEKO Congress, Helsinki, Finland, 2017.

[18] M. Kreuzer, "Die Schnittstelle zwischen Aufnehmer und Messverstärker bei Gleichspannung und Trägerfrequenzspeisung”, Messtechnische Briefe (HBM) issue 26, Darmstadt, Germany, pp. 42-48, 1990.

[19] M. Kreuzer, "Ein programmierbares PräzisionsMeßgerät der Ge-nauigkeitsklasse 0,0005 und seine Anwendungen", Messtechnische Briefe (HBM) issue 16, Darmstadt, Germany, pp. 41-46, 1980.

[20] M. Schäck, "Improving the Characteristics of a High-Precision Measuring Amplifier by a Powerful Digital Signal Processing", Proceedings of XXII IMEKO World Congress, Belfast, United Kingdom, 2018. 